\title{
Calculation of gain and luminescence spectra of quantum-cascade laser structures taking into account asymmetric emission line broadening
}

\author{
D.V. Ushakov, V.K. Kononenko, I.S. Manak
}

\begin{abstract}
The energy levels, wave functions, and matrix elements of optical dipole transitions are calculated numerically for superlattice quantum-cascade structures. The effect of spectral broadening on the shape of emission spectra is estimated and semiphenomenological asymmetric profiles of emission line broadening are proposed. It is shown that the electroluminescence spectra well agree with the calculated spontaneous recombination spectra.
\end{abstract}

Keywords: quantum-cascade superlattice, gain, luminescence, line broadening profile, AlInAs-GaInAs heterostructure.

\section{Introduction}

The quantum-cascade structures serve as a base of semiconductor emitters and photodetectors of the mid- and farIR regions. They belong to unipolar periodic systems in which optical transitions and electrotransfer occur through miniband states [1,2]. The shape of the emission and photosensitivity bands of such structures are determined mainly by the broadening and overlapping of spectral lines.

In this work we consider various asymmetric profiles of emission line broadening in superlattice quantum-cascade structures and estimate their effect on the shape of the gain and spontaneous recombination spectra. The calculated emission spectra are compared with the observed electroluminescence spectra of heterostructures in the AlInAs-GalnAs system.

\section{Emission line broadening profiles}

When designing quantum-cascade laser structures and optimising their characteristics, one should take into account the broadening of intersubband transition lines, bearing in mind that the simulation results strongly depend on the form of the used spectral broadening functions. Most frequently, one uses symmetric functions with the Gaussian, Lorentzian, or exponential (see [3, 4]) profiles of line broadening. The functional dependences and halfheight widths of symmetric profiles are presented in Table 1. One can see from Fig. 1a that the Gaussian (G) curve falls most rapidly and the Lorentzian (L) profile falls most slowly. The exponential $\mathrm{U}$ and $\mathrm{E}$ curves lie between the $\mathrm{G}$ and $\mathrm{L}$ profiles, the $\mathrm{E}$ profile being flatter than $\mathrm{U}$. The numerical curves in Fig. 1 are calculated for identical emission line half-widths according to Table 1.

Experimental observations show that the spectral line broadening profile is asymmetric $[4,5]$. Such a profile can be obtained assuming that it is described by the function $C_{-} F_{1}(\Delta E)$ at at $\Delta E<0$ and by the function $C_{+} F_{2}(\Delta E)$

Table 1. Symmetric emission line broadening profiles.

\begin{tabular}{|c|c|c|}
\hline Broadening profile & Function form & Line half-width \\
\hline Lorentzian (L) & $F_{\mathrm{L}}\left(\Delta E, \gamma_{\mathrm{L}}\right)=\frac{1}{\pi} \frac{\gamma_{\mathrm{L}}}{\Delta E^{2}+\gamma_{\mathrm{L}}^{2}}$ & $\gamma_{\mathrm{L}}$ \\
\hline Gaussian (G) & $F_{\mathrm{G}}\left(\Delta E, \gamma_{\mathrm{G}}\right)=\frac{1}{\sqrt{\pi} \gamma_{\mathrm{G}}} \exp \left(-\frac{\Delta E^{2}}{\gamma_{\mathrm{G}}^{2}}\right)$ & $\gamma_{G} \sqrt{\ln 2}$ \\
\hline Exponential (U) [3] & $F_{\mathrm{U}}\left(\Delta E, \gamma_{\mathrm{U}}\right)=\frac{1}{\gamma_{\mathrm{U}}}\left[\exp \left(\frac{\Delta E}{2 \gamma_{\mathrm{U}}}\right)+\exp \right.$ & $\gamma_{U} \ln (3+2 \sqrt{2})$ \\
\hline Exponential (E) [4] & $F_{\mathrm{E}}\left(\Delta E, \gamma_{\mathrm{E}}\right)=\frac{2}{\pi \gamma_{\mathrm{E}}}\left[\exp \left(\frac{\Delta E}{\gamma_{\mathrm{E}}}\right)+\exp \right.$ & $\gamma_{\mathrm{E}} \ln (2+\sqrt{3})$ \\
\hline
\end{tabular}

D.V. Ushakov, I.S. Manak Belarussian State University, prosp. Nezavisimosti 4, 220030 Minsk, Belarus; e-mail: UshakovDV@bsu.by,manak@bsu.by;

V.K. Kononenko B.I. Stepanov Institute of Physics, National Academy of Sciences of Belarus, prosp. Nezavisimosti 70, 220072 Minsk, Belarus

Received 8 July 2009

Kvantovava Elektronika 40 (3) 195-198 (2010)

Translated by M.N. Basieva at $\Delta E>0$. Joining the functions at zero $\left[C_{-} F_{1}(0)=\right.$ $\left.C_{+} F_{2}(0)\right]$ and taking into account the normalisation condition $\left(C_{-}+C_{+}=2\right)$, we can determine the $C_{-}$and $C_{+}$ coefficients. A combined model of the emission line broadening, in which, at the same broadening parameters, the profile is described by a Lorentzian at $\Delta E<0$ and by a Gaussian at $\Delta E>0$, was proposed in [6].

Table 2 presents the calculated functional dependences of asymmetric LG, LU, and LE emission line broadening 

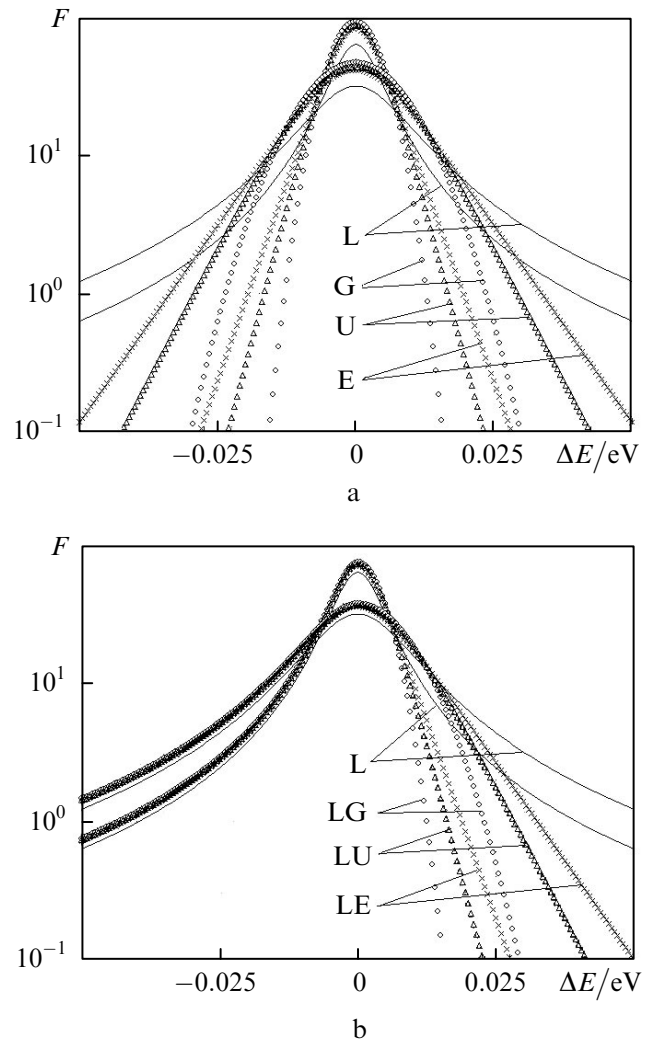

Figure 1. Spectral distributions $F(\Delta E)$ of symmetric (a) and asymmetric (b) line broadening for the broadening parameter $\gamma_{\mathrm{L}}=5$ and $10 \mathrm{meV}$.

profiles. In all the cases, we assumed that the region at $\Delta E<0$ is described by a Lorentzian and that the line widths at half maximum are identical. Table 2 also present the DA profile obtained in [7] based on the perturbation theory for a multiparticle system with the Coulomb interaction. The spectral curves of asymmetric line broadening are shown in Fig. 1b. One can see that, at the long-wavelength wing at $\Delta E<0$, all the curves exponentially decay and coincide with the $\mathrm{L}$ profile, while these curves at the short-wavelength wing at $\Delta E>0$ intersect with each other and exponentially decay according to the $\mathrm{G}, \mathrm{U}$, and $\mathrm{E}$ functions.

\section{Calculation of emission characteristics}

The dependence of the gain $g$ for intersubband transitions on the frequency of light $v$ in the multilevel approximation by neglecting the emission line broadening has the form of a delta function

$$
\begin{aligned}
& g_{n m}(v) \sim h v\left|z_{n m}\right|^{2} \int \mathrm{d} E_{\mathrm{f}}\left[f\left(E_{\mathrm{i}}\left(k_{\mathrm{f}}\right)\right)-f\left(E_{\mathrm{f}}\right)\right] \\
& \times \delta\left[E_{\mathrm{i}}\left(k_{\mathrm{f}}\right)-E_{\mathrm{f}}-h v\right] .
\end{aligned}
$$

Let us introduce the broadening profile according to $[5,6,8]$. Then

$$
\begin{aligned}
& g_{n m}(v) \sim\left|z_{n m}\right|^{2} \int \mathrm{d} E_{\mathrm{if}} E_{\mathrm{if}} F\left(h v-E_{\mathrm{if}}\right) \int \mathrm{d} E_{\mathrm{f}}\left[f\left(E_{\mathrm{i}}\left(k_{\mathrm{f}}\right)\right)\right. \\
& \left.-f\left(E_{\mathrm{f}}\right)\right] \delta\left[E_{\mathrm{i}}\left(k_{\mathrm{f}}\right)-E_{\mathrm{f}}-E_{\mathrm{if}}\right] .
\end{aligned}
$$

After integration in (2), we will have the final expression for the gain $g(v)$ for intrasubband transitions in the multilevel approximation [9]:

$$
\begin{aligned}
g(v) & =\sum_{n} \sum_{m} g_{n m}(v)=\frac{e^{2}}{\hbar^{3} \varepsilon_{0} c n_{\mathrm{r}} d} \sum_{n} \sum_{m} m_{\mathrm{f} m}\left|z_{n m}\right|^{2} \\
& \times \int_{E_{\mathrm{f} m}}^{\infty} \mathrm{d} E_{\mathrm{f}}\left(E_{\mathrm{i}}-E_{\mathrm{f}}\right) F\left[h v-\left(E_{\mathrm{i}}-E_{\mathrm{f}}\right), \gamma\right] \\
& \times\left[f\left(E_{\mathrm{i}}-F_{n}\right)-f\left(E_{\mathrm{f}}-F_{m}\right)\right],
\end{aligned}
$$

where $E_{\mathrm{i}}=\left(E_{\mathrm{f}}-E_{\mathrm{f} m}\right)\left(m_{\mathrm{f} m} / m_{\mathrm{i} n}\right)+E_{\mathrm{i} n} ; z_{n m}=\int \psi_{n}^{*} z \psi_{m} \mathrm{~d} z$ is the dipole transition matrix element; $F_{n}$ and $F_{m}$ are the quasi-Fermi levels in the $n$ and $m$ minibands with energies $E_{\mathrm{i} n}$ and $E_{\mathrm{f} m}$ and effective masses $m_{\mathrm{i} n}$ and $m_{\mathrm{f} m} ; d$ is the thickness of the quantum-cascade structure; $n_{\mathrm{r}}$ is the refractive index; and $\varepsilon_{0}$ is the electric constant. Summation

\begin{tabular}{|c|c|c|}
\hline Broadening profile & Function form & Broadening parameter \\
\hline Lorentz-Gauss (LG) & $\begin{array}{l}F_{\mathrm{LG}}\left(\Delta E, \gamma_{\mathrm{L}}\right)=\left\{\begin{array}{l}C_{-} F_{\mathrm{L}}\left(\Delta E, \gamma_{\mathrm{L}}\right), \Delta E \leqslant 0, \\
C_{+} F_{\mathrm{G}}\left(\Delta E, \gamma_{\mathrm{G}}\right), \Delta E \geqslant 0\end{array}\right. \\
C_{-}=\frac{2 \sqrt{\pi \ln 2}}{1+\sqrt{\pi \ln 2}}, \quad C_{+}=\frac{2}{1+\sqrt{\pi \ln 2}}\end{array}$ & $\gamma_{\mathrm{G}}=\frac{\gamma_{\mathrm{L}}}{\sqrt{\ln 2}}$ \\
\hline Lorentz-Unger (LU) & $\begin{array}{l}F_{\mathrm{LU}}\left(\Delta E, \gamma_{\mathrm{L}}\right)=\left\{\begin{array}{l}C_{-} F_{\mathrm{L}}\left(\Delta E, \gamma_{\mathrm{L}}\right), \Delta E \leqslant 0, \\
C_{+} F_{\mathrm{U}}\left(\Delta E, \gamma_{\mathrm{U}}\right), \Delta E \geqslant 0\end{array}\right. \\
C_{-}=\frac{2 \pi \ln (3+2 \sqrt{2})}{4+\pi \ln (3+2 \sqrt{2})}, \quad C_{+}=\frac{8}{4+\pi \ln (3+2 \sqrt{2})}\end{array}$ & $\gamma_{\mathrm{U}}=\frac{\gamma_{\mathrm{L}}}{\ln (3+2 \sqrt{2})}$ \\
\hline Lorentz-Eliseev (LE) & $\begin{array}{l}F_{\mathrm{LE}}\left(\Delta E, \gamma_{\mathrm{L}}\right)=\left\{\begin{array}{l}C_{-} F_{\mathrm{L}}\left(\Delta E, \gamma_{\mathrm{L}}\right), \Delta E \leqslant 0, \\
C_{+} F_{\mathrm{E}}\left(\Delta E, \gamma_{\mathrm{E}}\right), \Delta E \geqslant 0\end{array}\right. \\
C_{-}=\frac{2 \ln (2+\sqrt{3})}{1+\ln (2+\sqrt{3})}, C_{+}=\frac{2}{1+\ln (2+\sqrt{3})}\end{array}$ & $\gamma_{\mathrm{E}}=\frac{\gamma_{\mathrm{L}}}{\ln (2+\sqrt{3})}$ \\
\hline Drozd-Afonenko (DA) & $F_{\mathrm{A}}\left(\Delta E, \gamma_{\mathrm{A}}\right)=\frac{1}{\pi} \frac{\gamma_{\mathrm{A}}}{\gamma_{\mathrm{A}}^{2}+\Delta E^{2}} \frac{2}{1+\exp (\Delta E / k T)}$ & $\gamma_{\mathrm{A}}=\gamma_{\mathrm{L}}$ \\
\hline
\end{tabular}
in (3) is performed over all quantum numbers of the initial $(n)$ and final $(m)$ states, for which $E_{\mathrm{i} n}-E_{\mathrm{f} m}>0$.

It is known that the spontaneous recombination rate $r_{\mathrm{sp}}(h v)$ and the gain $g(v)$ for the conduction-valence band transitions in semiconductors satisfy the universal relation.

Table 2. Asymmetric emission line broadening profiles. 
Table 3. Parameters of the band structure of semiconductors.

\begin{tabular}{|c|c|c|c|c|c|c|c|c|c|c|c|}
\hline Compound & $m_{\mathrm{c}} / m_{\mathrm{e}}$ & $\gamma_{1}$ & $\gamma_{2}$ & $\gamma_{3}$ & $E_{p} / \mathrm{eV}$ & $\Delta S_{0} / \mathrm{meV}$ & $E_{g 0} / \mathrm{eV}$ & $E_{g 300} / \mathrm{eV}$ & $\alpha / \mathrm{meV} \mathrm{K}^{-1}$ & $\beta / \mathrm{K}$ & $F$ \\
\hline$\overline{\mathrm{GaAs}}$ & 0.067 & 6.98 & 2.06 & 2.93 & 28.8 & 341 & 1.519 & 1.42 & 0.5405 & 204 & -1.938 \\
\hline AlAs & 0.15 & 3.76 & 0.82 & 1.42 & 21.1 & 280 & 3.099 & 3.0 & 0.885 & 530 & -0.477 \\
\hline InAs & 0.026 & 20.0 & 8.5 & 9.2 & 21.5 & 390 & 0.417 & 0.354 & 0.276 & 93 & -2.896 \\
\hline $\mathrm{b}$ (GaInAs) & 0.0091 & 0 & 0 & 0 & -1.48 & 150 & 0.477 & 0 & 0 & 0 & 1.77 \\
\hline b (AlInAs) & 0 & 0 & 0 & 0 & -4.81 & 150 & 0.7 & 0 & 0 & 0 & -4.44 \\
\hline $\mathrm{Ga}_{0.47} \mathrm{In}_{0.53} \mathrm{As}$ & 0.041 & 13.88 & 5.47 & 6.25 & 25.3 & 330 & 0.816 & 0.735 & 0.4 & 145 & -2.886 \\
\hline $\mathrm{Al}_{0.48} \mathrm{In}_{0.52} \mathrm{As}$ & 0.073 & 12.2 & 4.81 & 5.47 & 22.51 & 300 & 1.530 & 1.445 & 0.568 & 303 & -0.627 \\
\hline
\end{tabular}

Note: $m_{\mathrm{c}}$ is the effective electron mass; $\gamma_{1}, \gamma_{2}, \gamma_{3}$ are the Luttinger parameters; $E_{p}$ is the energy related to the Kane matrix element of interband transitions; $\Delta S_{0}$ is the spin-orbit splitting energy; $E_{\mathrm{g}}$ is the band gap width; $\alpha$ and $\beta$ are the Varshni parameters for calculating the temperature dependence of the band gap width; $F$ is the parameter characterising the interaction of the conduction band with higher-lying energy bands.

For unipolar quantum-cascade lasers, the universal relation for intersubband transitions has the form [9]

$$
\begin{aligned}
& r_{\mathrm{sp}}(h v)=v_{\mathrm{gr}} \rho(h v) \sum_{n} \sum_{m} g_{n m}(v) \\
& \times\left[1-\exp \frac{\left(E_{n}-E_{m}\right)-\left(F_{n}-F_{m}\right)}{k T}\right]^{-1},
\end{aligned}
$$

where $\rho(h v)=(h v)^{2} n_{\mathrm{r}}^{2} /\left(\pi^{2} c^{2} \hbar^{3} v_{\mathrm{gr}}\right)$ is the density of electromagnetic modes and $v_{\mathrm{gr}}$ is the velocity of light in the crystal.

\section{Discussion of results}

The energy and the shape of wave functions for an arbitrarily shaped potential well were found by the $\boldsymbol{k} \cdot \boldsymbol{p}$ method in the extended Bastard model by solving the stationary Schrödinger equation $[10,11]$. The band structure parameters for the ternary compounds $\mathrm{A}_{x} \mathrm{~B}_{1-x} \mathrm{C}$ were approximated by experimental and theoretical data for binary compounds $\mathrm{AB}$ according to [12] using the quadratic approximation parameter $b_{\mathrm{ABC}}$,

$$
D_{\mathrm{ABC}}(x)=x D_{\mathrm{AC}}+(1-x) D_{\mathrm{BC}}-x(1-x) b_{\mathrm{ABC}} .
$$

The tabular band structure parameters for GaAs, AlAs, and InAs, as well as the data extrapolated by formula (5) for the $\mathrm{Ga}_{0.47} \operatorname{In}_{0.53}$ As and $\mathrm{Al}_{0.48} \mathrm{In}_{0.52}$ As compounds are listed in Table 3.

Figures 2 and 3 show the results of numerical calculations of the band structure, energy levels, wave functions, dipole transition matrix elements, and gain and emission spectra of the $\mathrm{Al}_{0.48} \mathrm{In}_{0.52} \mathrm{As}-\mathrm{Ga}_{0.47} \mathrm{In}_{0.53} \mathrm{As}$ heterostructure. The structure layers have the thicknesses (in nanometers, from left to right) $4.4 / \mathbf{1 . 7} / 0.9 / \mathbf{5 . 3} / 1.1 /$ 5.2/ $1.2 / 4.7 / 1.3 / 4.2 / 1.5 / 3.9 / 1.6 / 3.4 / 1.8 / 3.1 / 2.1 /$ $\underline{\mathbf{2 . 8} / 2.5 / 2.7 / 3.2 / 2.7 / 3.6 / 2.5}$. In the sequence of layers, the quantum wells of $\mathrm{Ga}_{0.47} \mathrm{In}_{0.53}$ As are given in bold and the layers doped with $\mathrm{Si}$ with a concentration of $1.5 \times 10^{17} \mathrm{~cm}^{-3}$ are underlined [13]. In Fig. 2a, the square moduli of wave functions corresponding to the $N$ th period are shown by thick lines, and thin lines correspond to the $(N-1)$ th period. In calculations, the height $\Delta E_{\mathrm{c}}$ of potential barriers in the conduction band was taken to be $0.51 \mathrm{eV}$. The effective masses of current carriers $m_{\mathrm{c}}$ were calculated to be $0.073 m_{\mathrm{e}}$ for the $\mathrm{Al}_{0.48} \mathrm{In}_{0.52} \mathrm{As}$ barrier layers and $0.041 m_{\mathrm{e}}$ for the $\mathrm{Ga}_{0.47} \mathrm{In}_{0.53}$ As quantum wells.

The occupation of energy levels and the corresponding quasi-Fermi levels were found by numerically solving the system of balance equations [14]. The calculated emission
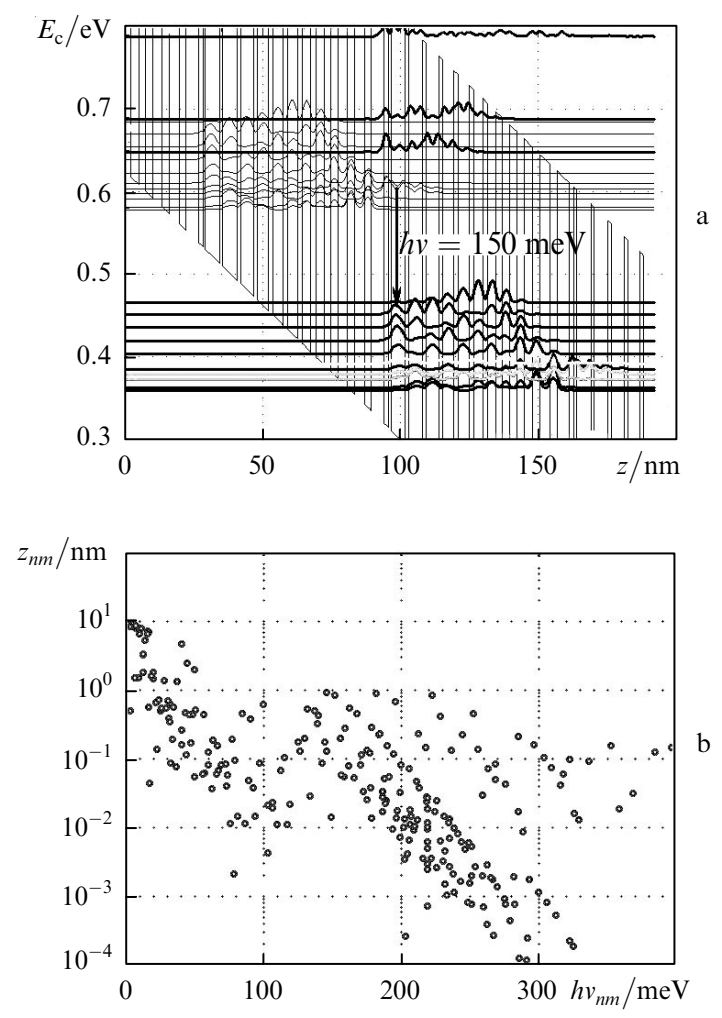

Figure 2. Conduction band diagram $E_{\mathrm{c}}(z)$ and square moduli of electron wave functions (a) and dipole transition matrix elements $z_{n m}$ (b) for the $\mathrm{Al}_{0.48} \mathrm{In}_{0.52} \mathrm{As}-\mathrm{Ga}_{0.47} \mathrm{In}_{0.53} \mathrm{As}$ quantum-cascade structure in an electric field with the strength $E=3.25 \times 10^{4} \mathrm{~V} \mathrm{~cm}^{-1} ; h v_{n m}=E_{n}-E_{m}$.

spectra are shown in Fig. 3. One can see that the absorption of light dominates at low electric field strengths (below $\left.2.0 \times 10^{4} \mathrm{~V} \mathrm{~cm}^{-1}\right)$. With increasing the excitation strength $E$ from $2.1 \times 10^{4}$ to $3.4 \times 10^{4} \mathrm{~V} \mathrm{~cm}^{-1}$, the gain maximum shifts from 115 to $150 \mathrm{meV}$. With a further increase in the electric field strength, one observes a shift in laser levels, the gain decreases, but new peaks appear in the shortwavelength region at 190 and $220 \mathrm{meV}$. In this quantumcascade structure, two-phonon resonance (when the distance between the lower laser level of a preceding cascade and the upper laser level of the next cascade is equal to the double optical phonon energy) occurs at $E=3.25 \times 10^{4} \mathrm{~V} \mathrm{~cm}^{-1}$. In this case, the calculated threshold current density $J=2.19 \mathrm{kA} \mathrm{cm}^{-2}$ well agrees with experimental data [13].

The emission characteristics of the superlattice quantumcascade structures were calculated using different models of emission line broadening. For the Lorentzian emission line 


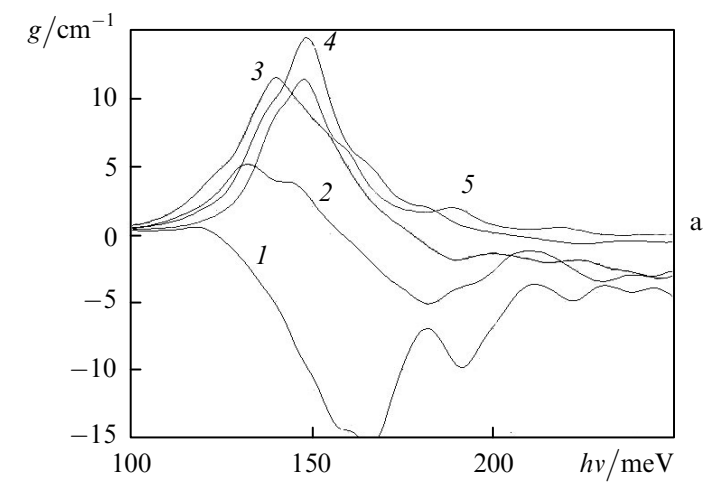

$r_{\mathrm{sp}} / 10^{19} \mathrm{~cm}^{-2} \mathrm{eV}^{-1} \mathrm{~s}^{-1}$

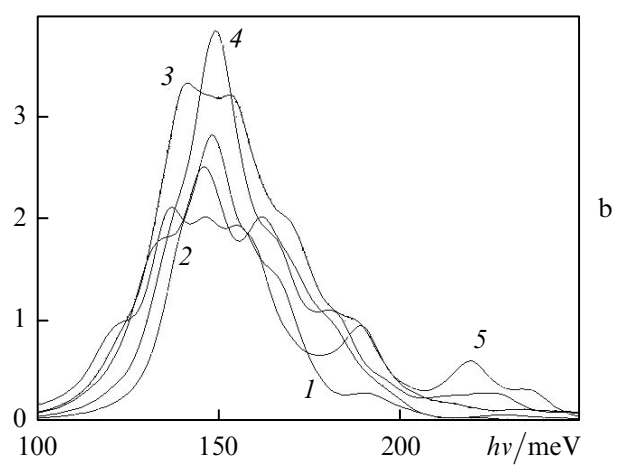

Figure 3. Gain $g(v)$ (a) and spontaneous emission $r_{\mathrm{sp}}(h v)$ (b) spectra of a quantum-cascade structure calculated taking into account the LE broadening profile at $E=2.0$ (1), 2.4 (2), 2.8 (3), 3.4 (4), and $4.0 \times$ $10^{4} \mathrm{~V} \mathrm{~cm}^{-1}(5) ; \gamma_{\mathrm{L}}=7 \mathrm{meV} ; T=300 \mathrm{~K}$.
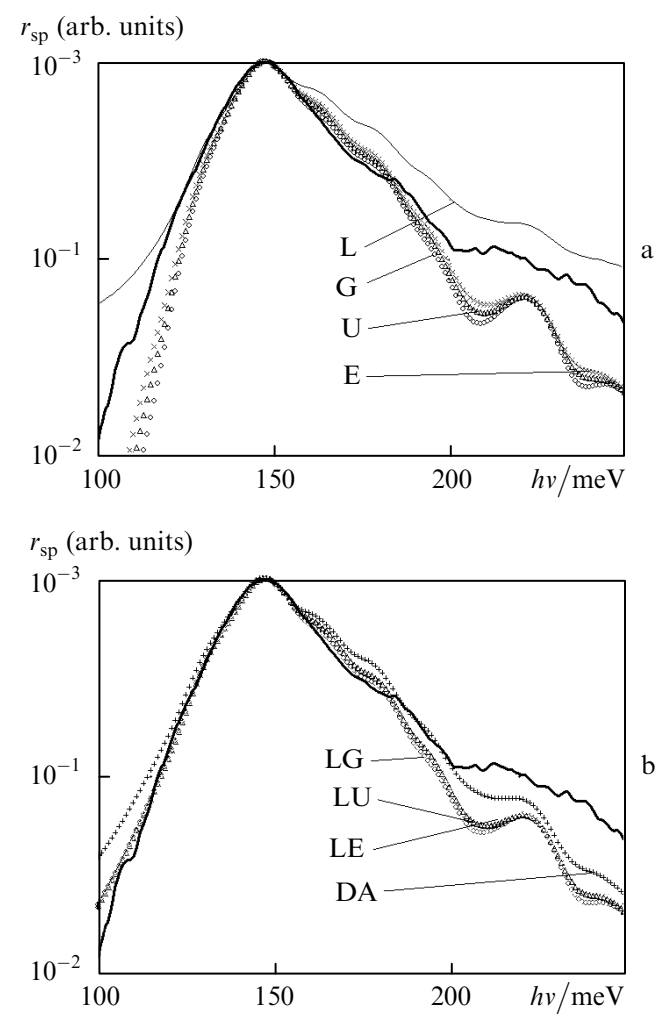

Figure 4. Electroluminescence spectra taken from [13] (thick curve) in comparison with the spontaneous emission spectra of a superlattice quantum-cascade structure calculated for symmetric (a) and asymmetric (b) broadening profiles with the broadening parameter $\gamma_{\mathrm{L}}=7 \mathrm{meV}$; $E=3.25 \times 10^{4} \mathrm{~V} \mathrm{~cm}^{-1}, T=300 \mathrm{~K}$. profile, we used the broadening parameter $\gamma_{\mathrm{L}}$, while the other broadening parameters were found according to Tables 1 and 2. For comparison, Fig. 4 shows the calculated emission spectra and the luminescence spectra measured at $T=300 \mathrm{~K}$ [13]. The optical transitions in the considered structure lie in the wavelength region of $\sim 8.45 \mu \mathrm{m}$ $(150 \mathrm{meV})$. As follows from Fig. 4, the asymmetric LG, LU, LE, and DA line broadening profiles perfectly describe the experimental spectra. The Lorentzian model is the worst for describing the emission spectra.

\section{Conclusions}

Thus, we have numerically calculated the gain and spontaneous emission spectra of quantum-cascade structures. The proposed semiphenomenological expressions for asymmetric emission line broadening allow one to adequately describe the emission bands for intersubband transitions. It is shown that the calculated emission spectra well agree with experimental data.

Acknowledgements. The authors thank A.A. Afonenko for discussion of the results. This work was supported by the Belarussian Republican Foundation for Basic Research (Grant No. F08R-119).

\section{References}

1. Kazarinov R.F., Suris R.A. Fiz. Tekhn. Polupr., 5, 797 (1971) [Sov. Phys. Semicond., 5, 707 (1971)].

2. Faist J., Capasso F., Sivco D.L., Sirtori C., Hutchinson A.L., Cho A.Y. Science, 264, 553 (1994).

3. Unger K. Phys. Stat. Sol. B, 149, K141 (1988).

4. Eliseev P.G., Akimova I.V. Fiz. Tekhn. Polupr., 32, 478 (1998) [Semiconductors, 32, 428 (1998)].

5. Yamanishi M., Lee Y. IEEE J. Quantum Electron., 23, 367 (1987).

6. Kononenko V.K., Manak I.S., Nalivko S.V., Shevtsov V.A., Shulyaev D.S. Zh. Prikl. Spectrosk., 64, 221 (1997) [J. Appl. Spectrosc., 64, 234 (1997)].

7. Drozd A.N., Afonenko A.A. Zh. Prikl. Spectrosk., 74, 642 (2007) [J. Appl. Spectrosc., 74, 642 (2007)].

8. Asada M. IEEE J. Quantum Electron., 25, 2019 (1989).

9. Ushakov D.V., Manak I.S. Opt. Spectrosk., 104, 847 (2008) [Opt. Spectrosc., 104, 767 (2008)].

10. Yoo K.H., Ram-Mohan L.R., Nelson D.F. Phys. Rev. B, 39, 12808 (1989).

11. Sirtori C., Capasso F., Faist J., Scandolo S. Phys. Rev. B, 50, 8663 (1994).

12. Vurgaftman I., Meyer J.R., Ram-Mohan L.R. J. Appl. Phys., 89, 5815 (2001).

13. Wittmann A., Gresch T., Gini E., Hvozdara L., Hoyler N., Giovannini M., Faist J. IEEE J. Quantum Electron., 44, 36 (2008).

14. Ushakov D.V., Manak I.S. Zh. Prikl. Spectrosk., 74, 801 (2007) [J. Appl. Spectrosc., 74, 892 (2007)]. 